\title{
The benefit of assisted and unassisted eco-driving for electrified powertrains
}

\author{
Xingda Yan, Craig K. Allison, James M. Fleming ${ }^{\ddagger}$, \\ Neville A. Stanton and Roberto Lot
}

\begin{abstract}
Eco-driving assistance systems that encourage drivers to engage in fuel-saving behaviour are effective at improving energy-efficiency, with recent research directed towards incorporating predictive models of energy losses in these systems to optimise recommendations. In this paper we evaluate a predictive eco-driving assistance system on three powertrains: a combustion engine-driven vehicle, a parallel hybrid electric vehicle, and a battery electric vehicle. In each case, energy consumption is found by applying a quasi-static model to driving simulator data for a simulated route including urban, rural, and highway sections. We find that both assisted and unassisted eco-driving has a beneficial effect in all cases, with the assistance system leading to reductions in energy usage of $6.1 \%, 15.9 \%$ and $16.6 \%$ for the combustion engine, hybrid electric, and battery electric powertrains respectively.
\end{abstract}

Index Terms-Eco-Driving; Driver Assistance; Electric Vehicles; Hybrid Vehicles; Fuel-efficient driving;

\section{INTRODUCTION}

Motivated by climate change and the drive for sustainability, recent years have seen a great increase in measures to improve the energy efficiency of personal transportation. For combustion engine driven vehicles this lowers $\mathrm{CO}_{2}$ emissions, while for plug-in electric vehicles it reduces charging costs and increases range. Eco-driving, the modification of driving behaviour to save fuel and energy, is an efficacious method to reduce vehicle energy usage [1] that nonetheless can have limited effectiveness in practice due to difficulty in getting drivers to maintain energy-efficient behaviour longterm [2]. One suggestion to improve the uptake and retainment of eco-driving behaviour is to provide real-time feedback on a driver's energy efficiency through a visual [3] or auditory or haptic [4] humanmachine interface (HMI). This real-time feedback may be augmented with predictive or feedforward information on the behaviour of traffic and traffic signals to improve energy saving beyond what can be achieved by the driver alone [5].

\footnotetext{
$\ddagger$ Corresponding author: J.Fleming @lboro.ac.uk
}

A possible complication of eco-driving efforts is a trend towards electrification of vehicle powertrains [6], driven itself by the persuit of greater energyefficiency. Eco-driving recommendations such as gentle acceleration, late upshifting of gears and avoidance of braking [1] were developed for combustion engine driven vehicles. Electric powertrains can regenerate energy when braking and typically use an electric motor and a fixed reduction gear in place of a combustion engine and gearbox, leading to different optimal operating points for energyefficiency. The effect of driving style on energyefficiency of electric vehicles has been studied, and eco-driving behaviours shown to be effective for them [7]. These eco-driving behaviours may need to be adapted for electric vehicles, supporting the use of either specific driver training or assistance systems [8]. From a user perspective, a major difference in electrified powertrains is deceleration and energy recovery through regenerative braking. Although most drivers quickly learn to interact with such systems, a minority report difficulties [9]. Effective use of regenerative braking may need to be learnt, and it has been demonstrated that both drivers with experience of electric vehicles and those that have undergone specific training achieve greater energy efficiency [10]. From a technical standpoint, eco-driving optimisations have been developed for electric vehicles [11], and for hybrid vehicles [12], which may also use vehicle-infrastructure communication to provide recommendations.

In this work, we evaluate the efficacy on different powertrains of a recently-developed eco-driving assistance system incorporating a predictive model of vehicle energy losses and, novelly, driver preferences in following and cornering situations [13]. This is carried out by calculating energy usage for velocity profiles obtained from human participants in a driving simulator at the University of Southampton, UK. This assistance system provides speed recommendations based on a multiobjective 
optimisation of minimising energy loss and respecting typical driver preferences on vehicle speed, following distance, and cornering speed. This allows it to gently 'coach' drivers into natural, yet more energy-efficient behaviour. Fuel and/or electrical energy usage may be calculated after simulation using models of conventional, hybrid electric, and electric powertrains. The objective is to determine if the developed driver assistance system is effective across different powertrains, comparing it with normal driving and unassisted eco-driving in each case. Accordingly, we consider the following two hypotheses:

1) That the eco-driving assistance system will show improvements in energy-efficiency across all three powertrain types.

2) Improvements in energy-efficiency will be comparable to those resulting from deliberate, unassisted eco-driving.

We do not include an in-depth description of the assistance system and optimisation itself or report measures of driving style and cognitive workload. Instead, we direct readers to the authors' other works [13], [14] to cover these aspects.

\section{POWERTRAin Models}

To evaluate energy consumption, we used quasistatic [15] models of the three powertrains in Fig. 1. These models were developed in Simulink and validated as part of a wider research project [16]. For each participant, the velocity of the modelled vehicle was controlled to track the velocity profile of the participants in the driving simulator study using a Proportional-Integral-Derivative (PID) feedback controller tuned to apply force to give less than $0.01 \mathrm{~m} / \mathrm{s}$ mean-square error in velocity with no overshoot. Components of the three powertrains were sized so that each had a total power output of $150 \mathrm{~kW}$, to match the characteristics of the driving simulation. All three models considered equal driving energy losses due to rolling resistance and aerodynamic drag. Hence, in each case the overall driving force $F$ on the wheels was:

$$
F=m a_{x}+\frac{1}{2} \rho A_{f} C_{d} v^{2}+C_{r r} m g
$$

where $m$ is the vehicle mass, $\rho$ the air density, $A_{f}$ the frontal area, $C_{d}$ the coefficient of drag, and $C_{r r}$ the coefficient of rolling resistance. Once the force required to follow the velocity profile was

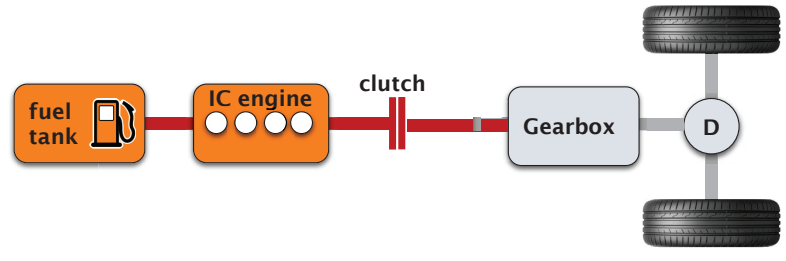

(a) Conventional

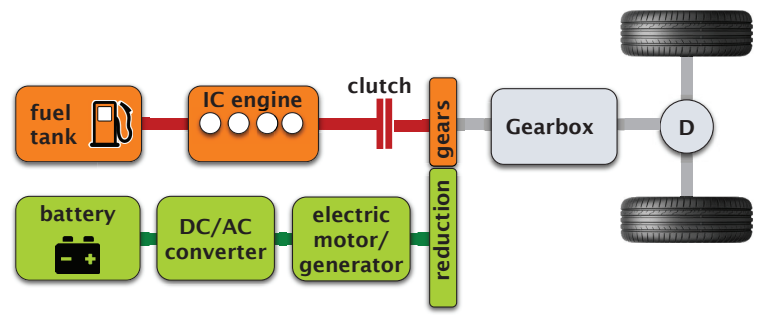

(b) Parallel hybrid

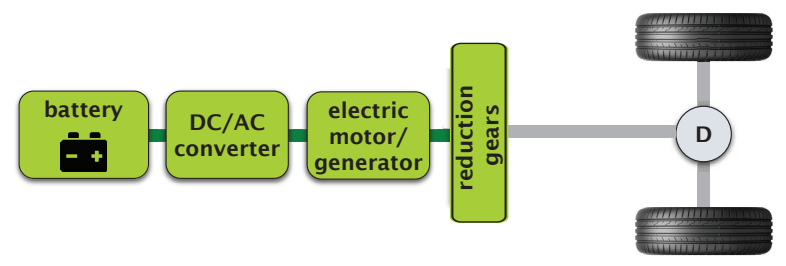

(c) Electric

Fig. 1: Powertrains considered in the study

TABLE I: Vehicle parameters

\begin{tabular}{ll|ll} 
Parameter & Value & Parameter & Value \\
\hline$m$ & $1500 \mathrm{~kg}$ & $\rho$ & $1.225 \mathrm{~kg} / \mathrm{m}^{3}$ \\
$g$ & $9.81 \mathrm{~N} / \mathrm{kg}$ & $C_{r r}$ & 0.008 \\
$C_{d}$ & 0.4 & $r_{w}$ & $0.3 \mathrm{~m}$ \\
$A_{f}$ & $2 m^{2}$ & &
\end{tabular}

found, maps of fuel and electrical energy usage were evaluated using linear interpolation to find instantaneous fuel/energy consumption, which was integrated over the entire journey. Values of the parameters used in the study are given in Table I.

\section{A. Conventional powertrain}

For the conventional internal combustion engine (ICE) powertrain (Fig. 1a), the wheels are driven by a $150 \mathrm{~kW}$ gasoline engine via the gearbox. Gear changes are automatic according to a typical twoparameter gear shifting schedule. Total driving force $F$ is the sum of force caused by the engine and the hydraulic brakes. Fuel consumption is modelled as a lookup table with two inputs: crankshaft speed and engine torque, which are related to vehicle speed 


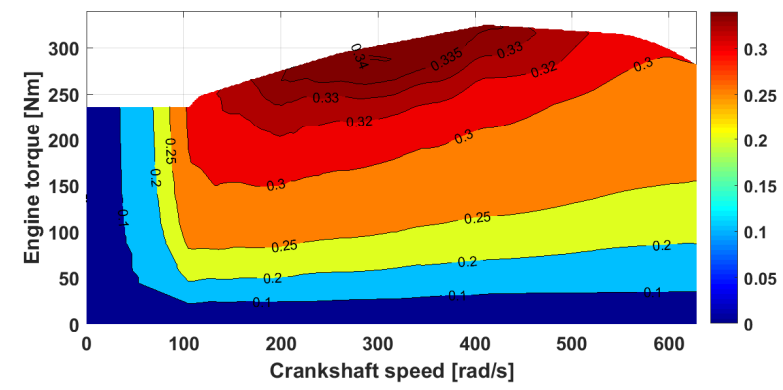

Fig. 2: Efficiency Map of the IC Engine

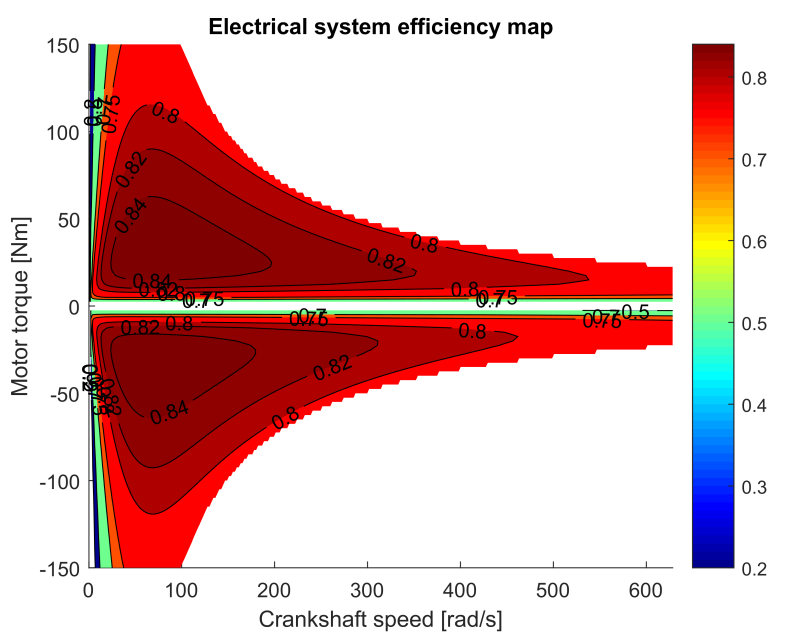

Fig. 3: Efficiency Map of the electric traction motor

$v$ and driving force $F$ via the transmission. The efficiency map used for the ICE was obtained from $1 \mathrm{~d}$ fluid dynamics simulations in Ricardo Wave and is given in Fig. 2. As is typical for a gasoline engine, peak efficiency occurs at high torque and moderate RPM, and efficiency decreases rapidly below 100 $\mathrm{rad} / \mathrm{s}$ due to clutch slip.

\section{B. Parallel hybrid powertrain}

The second powertrain considered is the parallel hybrid electric vehicle (HEV) (Fig. 1b). In addition to the ICE, this includes a $15 \mathrm{~kW}$ electric traction motor, which is coupled to the driving shaft via a fixed reduction gear. Hence, the driving force $F$ is provided by three sources: the engine, the traction motor and the hydraulic brakes. The fuel consumption model is identical to that used for the conventional powertrain, while the electrical traction system is modelled in a similar manner using the efficiency map (Fig. 3). The performance of the HEV depends on the energy management strategy used to control the power split between these sources. In this paper, the well-known equivalent consumption minimization strategy (ECMS) [17] was used, which is optimal if the battery charge is equal at the start and end of the drive. The equivalence factor used was tuned to ensure this charge-sustaining behaviour, so that energy consumption may be evaluated considering fuel usage only.

\section{Battery electric powertrain}

The third powertrain is electric (Fig. 1c) and uses a similar electrical traction system to that in the parallel HEV. However, the battery and motor have been resized from $15 \mathrm{~kW}$ to $150 \mathrm{~kW}$ to match the performance of the ICE vehicle. For this powertrain, the driving force $F$ is provided by the motor and the hydraulic brakes. The motor may apply a negative force for regenerative braking, but this force is only applied on the front wheels so a proportion of braking energy is recovered when the driver uses the brake pedal in the simulator, with manual braking assumed for braking forces greater than can be provided by the electric motor.

\section{STUdy Method}

\section{A. Equipment}

The study was carried out in the fixed-base driving simulator at the University of Southampton, which comprises: a right-hand drive Land Rover Discovery; three projector screens in front of the vehicle with a 135-degree field of view; a single screen behind the vehicle showing an image visible in the rear-view mirror, and LCD displays on each side-view mirror to simulate the view there. A $21 \mathrm{~km}$ simulated route consisting of urban, rural and motorway sections was developed and driven using STISIM M500W wide-field-of-view driving simulation software, with the journey modelled on real-world roads around Southampton, UK. Engine noise in the simulator was turned off and the vehicle transmission was set to automatic, so that gear changes would not be required.

An additional speedometer was required to show participants their speed. This was implemented as a GUI application executed on a Microsoft Surface Pro tablet, which was placed behind the steering wheel of the car to replace the physical instrument cluster and display speed and RPM. The eco-driving assistance system considered is an eco-speedometer design that augments this speedometer with a green 
and yellow band to recommend a speed range. This recommendation is updated in real-time to provide a speed that approximately minimises energy losses due to rolling resistance, aerodynamic drag, and braking. The efficiency of the drivetrain is not taken into account, and the calculation of the recommended speed considers the current vehicle speed and acceleration, position and speed of any leading vehicle and the curvature of approaching corners. Further details on the visual HMI and the predictive speed recommendation may be found in [13].

\section{B. Study design}

Thirty-six participants (18 male, 18 female) were recruited, aged 19 to 71 years $(M=28.92$, $S D=12.82$ ). To acclimatize to the simulator, they completed two short practice drives, with the speed recommendation active for the second practice. The study used a repeated-measures design with one within-subjects independent variable, condition (control/eco/assisted), with the values:

- Control condition: The driver was instructed to drive as they normally would;

- Eco-driving condition: The driver was instructed to drive in order to save energy, after being shown a set of eco-driving tips: to avoid braking, to accelerate smoothly and gently, and to anticipate traffic flow;

- Assisted condition: The eco-driving assistance system displayed a band of recommended speeds on the vehicle speedometer. Drivers were informed that the band showed speeds for effective eco-driving, but were not explcitly instructed to follow the recommendation;

To reduce the influence of order effects on the results, the order that participants drove these conditions was fully counterbalanced with an equal number given each of the 6 possible permutations.

\section{Statistical analysis}

The significance level of 0.05 was chosen for all comparisons in the study. For each powertrain, a repeated-measures ANOVA was used to analyse the dependence of fuel/electric energy consumption on test condition. Tests of normality and sphericity were performed to check the assumptions of the ANOVA analysis. Where significant effects were observed, post hoc paired-sample t-tests were used
TABLE II: Descriptive statistics

\begin{tabular}{l|ll|ll|ll} 
& \multicolumn{2}{|c}{ Control } & \multicolumn{2}{c}{ Eco } & \multicolumn{2}{c}{ Assisted } \\
& M & SD & M & SD & M & SD \\
\hline Conv. $[1 / 100 \mathrm{~km}]$ & 7.02 & 0.53 & 6.40 & 0.43 & 6.59 & 0.33 \\
Hyb. $[1 / 100 \mathrm{~km}]$ & 5.93 & 0.81 & 4.89 & 0.71 & 4.99 & 0.36 \\
Elec. $[\mathrm{kWh} / 100 \mathrm{~km}]$ & 12.04 & 2.38 & 9.13 & 1.49 & 10.04 & 1.28
\end{tabular}

TABLE III: ANOVA results for test condition

\begin{tabular}{l|lll} 
Powertrain & $F$ & $p$ & $\eta_{p}^{2}$ \\
\hline Conventional & 29.8 & $<0.001^{*}$ & 0.467 \\
Hybrid & 44.5 & $<0.001^{*}$ & 0.567 \\
Electric & 41.9 & $<0.001^{*}$ & 0.552
\end{tabular}

to compare groups, applying a Bonferroni correction to account for multiple comparisons.

\section{RESULTS}

Descriptive statistics and ANOVA results are given in Tables II and III respectively. KolmogorovSmirnov tests indicated that residuals were not significantly different from a normal distribution of mean zero ( $p=0.52, p=0.36$, and $p=0.56$ for the conventional, hybrid and electric cases respectively). Mauchly's test indicated that the sphericity assumption of the ANOVA may be violated ( $p=0.026$, $p=0.055$ and $p=0.001$ ), hence GreenhouseGeisser corrections were applied. A significant main effect of test condition was observed in all cases. Accordingly, we performed post-hoc t-tests between different test conditions for each powertrain. The results of these post-hocs may be seen in Table IV. Differences were significant in all cases except between the eco-driving and assisted condition for the hybrid powertrain.

Boxplots of the fuel and electrical energy consumption of the three powertrains in the study are shown in Figs. 4-6, noting that the hybrid is operated in a charge-sustaining manner and its fuel usage represents the total energy use. Eco-driving lead to the lowest consumption in all three cases. The benefit ranged from a reduction of $8.83 \%$ for the conventional powertrain, to $24.2 \%$ in the electric powertrain, and the hybrid intermediate between these with a $17.5 \%$ reduction. Use of the driver assistance system also showed greater benefits for the electrified powertrains. For the conventional powertrain there was a $6.13 \%$ reduction in energy usage, but the hybrid and electric powertrains showed $15.9 \%$ and $16.6 \%$ reductions respectively.

Although it was not the objective of the study, we also note that the fuel consumption of the parallel 
TABLE IV: Post-hoc t-tests of fuel/energy usage

\begin{tabular}{l|lll} 
Condition Pair & mean diff. & $t$ & $p$ \\
\hline Conventional & & & \\
Control - Eco-driving & $0.621 / 100 \mathrm{~km}$ & 6.32 & $<0.001^{*}$ \\
Control - Assisted & $0.431 / 100 \mathrm{~km}$ & 5.64 & $<0.001^{*}$ \\
Assisted - Eco-driving & $0.191 / 100 \mathrm{~km}$ & 2.78 & $=0.009^{*}$ \\
Hybrid & & & \\
Control - Eco-driving & $1.051 / 100 \mathrm{~km}$ & 7.34 & $<0.001^{*}$ \\
Control - Assisted & $0.951 / 100 \mathrm{~km}$ & 8.24 & $<0.001^{*}$ \\
Assisted - Eco-driving & $0.101 / 100 \mathrm{~km}$ & 0.96 & $=0.343$ \\
Electric & & & \\
Control - Eco-driving & $2.90 \mathrm{kWh} / 100 \mathrm{~km}$ & 7.38 & $<0.001^{*}$ \\
Control - Assisted & $1.99 \mathrm{kWh} / 100 \mathrm{~km}$ & 6.19 & $<0.001^{*}$ \\
Assisted - Eco-driving & $0.91 \mathrm{kWh} / 100 \mathrm{~km}$ & 3.97 & $<0.001^{*}$
\end{tabular}

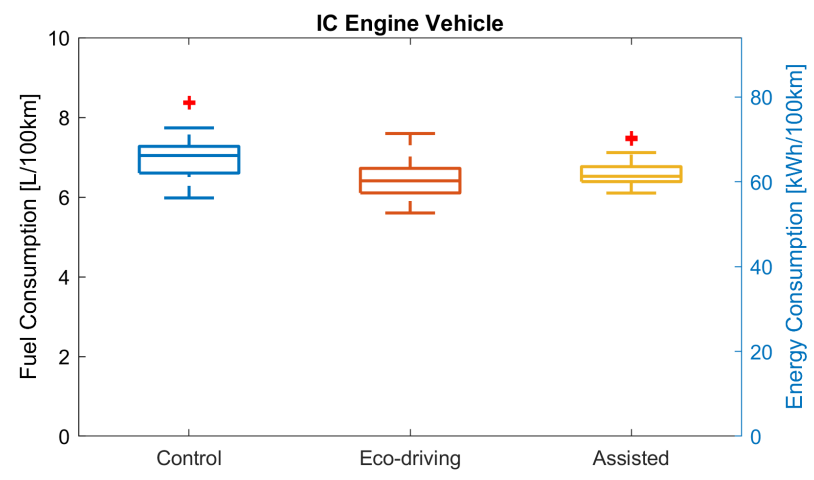

Fig. 4: Fuel usage of conventional powertrain

hybrid drivetrain was substantially $(15.5 \%-24.3 \%)$ lower than that of the conventional one for all trials, confirming that hybridisation still leads to benefits when eco-driving is used.

\section{Discussion}

In line with existing publications discussing ecodriving for conventional [1] and electric vehicles [7], the results confirm that the eco-driving advice to avoid braking, to accelerate smoothly and gently, and to anticipate traffic flow is useful to reduce energy consumption. As the same time-series data for velocity was used to evaluate each model, this also implies that generic eco-driving advice is efficacious to reduce drivers energy consumption in hybrid and battery electric vehicles. Indeed, proportionally greater reductions are observed for the two electrified powertrains than for the conventional one.

The results also add to the existing literature that supports the use of eco-driving assistance using visual feedback for both conventional [3], [5] and electric vehicles [8]. The present work extends this literature by demonstrating that this is also the case for assistance systems using predictive models of

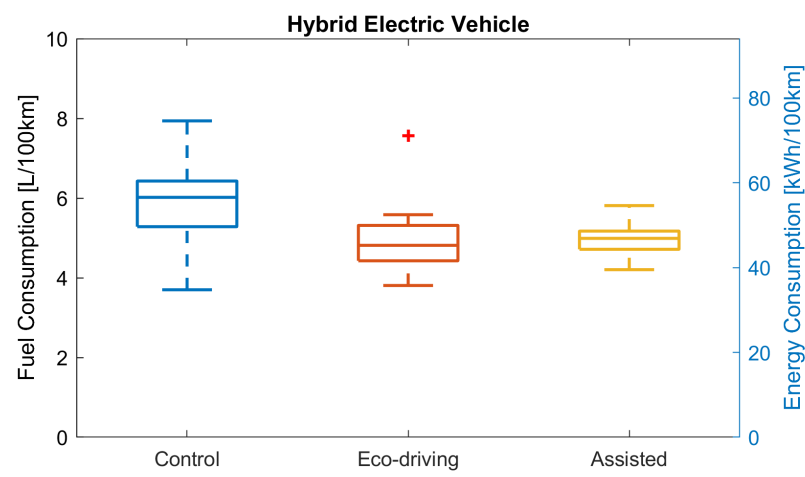

Fig. 5: Fuel usage of hybrid powertrain



Fig. 6: Energy usage of electric powertrain

lead vehicle motion and driver preferences [13], as considered here. However, the observation that the system is outperformed by deliberate eco-driving in the electric and conventional cases suggests that there is scope to improve its performance further.

\section{A. Limitations}

As the study was limited to evaluating velocity data from each participant using quasi-static models, simulated vehicle dynamics were the same for all powertrains. Hence, although overall power and acceleration were matched to the simulated vehicle in each case, the present study cannot account for differences in driving style resulting from characteristics of the powertrains, and in particular the possibility of one-pedal driving could not be considered in the electric vehicle case. The assumption underlying the present work is that the speed profile followed is similar regardless of which powertrain is used in the vehicle - if one-pedal driving were to significantly alter driving speed, acceleration or deceleration behaviour for the electric powertrain, our results may no longer be valid. As the study 
was carried out in a fixed-base simulator, driving style may also differ from real-world driving, for example due to the lack of lateral acceleration when cornering. It is also possible that features of the simulator itself may have affected participants' adopted driving styles, adapting their style after seeing the Land Rover Discovery, for example. We have not considered learning effects or changes over time, which is often an important aspect for ecodriving training and assistance systems.

An automatic transmission was assumed and the study did not incorporate the effect of drivers' gearchanging strategies. The driver assistance system used provided only a speed recommendation via a visual HMI and so the results may not generalise to other kinds of assistance system. Finally, the hybrid powertrain considered was a pre-transmission parallel hybrid, and so the results may not generalise to series or series-parallel powertrains.

\section{CONCLUSIONS}

We conducted a controlled trial of an eco-driving assistance system in a driving simulator, comparing it with normal driving and eco-driving. The simulator data was used to evaluate fuel and energy consumption for conventional, hybrid electric, and battery electric powertrains. This revealed that the eco-driving assistance system leads to reductions in energy usage, with proportionally greater reductions in the hybrid and electric cases, and similar improvements for both male and female drivers. Eco-driving outperformed the assistance system for the electric and conventional powertrains, suggesting that the assistance system could perhaps be improved in these cases. We also observed that the hybrid powertrain had lower fuel consumption than the conventional one in all trials, regardless of whether eco-driving was being used.

\section{ACKNOWLEDGEMENTS}

We are grateful for support of the UK Engineering and Physical Sciences Research Council (EPSRC) under grant EP/N022262/1 Green Adaptive Control for Future Interconnected Vehicles. The authors would also like to thank all study participants.

\section{REFERENCES}

[1] J. N. Barkenbus, "Eco-driving: An overlooked climate change initiative," Energy Policy, vol. 38, no. 2, pp. 762-769, 2010.
[2] B. Beusen et al., "Using on-board logging devices to study the longer-term impact of an eco-driving course," Transportation research part D: transport and environment, vol. 14, no. 7, pp. 514-520, 2009.

[3] A. Meschtscherjakov, D. Wilfinger, T. Scherndl, and M. Tscheligi, "Acceptance of future persuasive in-car interfaces towards a more economic driving behaviour," in Proceedings of the 1st International Conference on Automotive User Interfaces and Interactive Vehicular Applications. ACM, 2009, pp. 81-88.

[4] R. C. McIlroy, N. A. Stanton, L. Godwin, and A. P. Wood, "Encouraging eco-driving with visual, auditory, and vibrotactile stimuli," IEEE Trans. on Human-Machine Systems, vol. 47, no. 5, pp. 661-672, 2016.

[5] M. A. S. Kamal, M. Mukai, J. Murata, and T. Kawabe, "On board eco-driving system for varying road-traffic environments using model predictive control," in IEEE International Conference on Control Applications. IEEE, 2010, pp. 1636-1641.

[6] E. Tate, M. O. Harpster, and P. J. Savagian, "The electrification of the automobile: from conventional hybrid, to plug-in hybrids, to extended-range electric vehicles," SAE international journal of passenger cars-electronic and electrical systems, vol. 1, no. 2008-01-0458, pp. 156-166, 2008.

[7] M. Knowles, H. Scott, and D. Baglee, "The effect of driving style on electric vehicle performance, economy and perception," International Journal of Electric and Hybrid Vehicles, vol. 4, no. 3, pp. 228-247, 2012.

[8] I. Neumann, T. Franke, P. Cocron, F. Bühler, and J. F. Krems, "Eco-driving strategies in battery electric vehicle use-how do drivers adapt over time?" IET Intelligent Transport Systems, vol. 9, no. 7, pp. 746-753, 2015.

[9] P. Cocron, F. Bühler, T. Franke, I. Neumann, B. Dielmann, and J. F. Krems, "Energy recapture through decelerationregenerative braking in electric vehicles from a user perspective," Ergonomics, vol. 56, no. 8, pp. 1203-1215, 2013.

[10] M. Günther, N. Rauh, and J. F. Krems, "How driving experience and consumption related information influences eco-driving with battery electric vehicles-results from a field study," Transportation Research Part F: Traffic Psychology and Behaviour, vol. 62, pp. 435-450, 2019.

[11] W. Dib, A. Chasse, P. Moulin, A. Sciarretta, and G. Corde, "Optimal energy management for an electric vehicle in ecodriving applications," Control Engineering Practice, vol. 29, pp. 299-307, 2014.

[12] J. Hu, Y. Shao, Z. Sun, M. Wang, J. Bared, and P. Huang, "Integrated optimal eco-driving on rolling terrain for hybrid electric vehicle with vehicle-infrastructure communication," Transportation Research Part C: Emerging Technologies, vol. 68, pp. 228-244, 2016

[13] J. Fleming, X. Yan, C. Allison, N. Stanton, and R. Lot, "Driver modeling and implementation of a fuel-saving ADAS," in IEEE Conference on Systems, Man and Cybernetics, 2018.

[14] C. K. Allison, J. M. Fleming, X. Yan, R. Lot, and N. A. Stanton, "Adjusting the need for speed: Assessment of a visual interface to reduce fuel use," Ergonomics, 2020.

[15] E. Rishavy, S. Hamilton, J. Ayers, and M. Keane, "Engine control optimization for best fuel economy with emission constraints," SAE Technical Paper, Tech. Rep., 1977.

[16] X. Yan, J. Fleming, and R. Lot, "Modelling and energy management of parallel hybrid electric vehicle with air conditioning system," in 2017 IEEE Vehicle Power and Propulsion Conference. IEEE, 2017, pp. 1-5.

[17] G. Paganelli, G. Ercole, A. Brahma, Y. Guezennec, and G. Rizzoni, "General supervisory control policy for the energy optimization of charge-sustaining hybrid electric vehicles," JSAE review, vol. 22, no. 4, pp. 511-518, 2001. 